\title{
Modelling the overdiagnosis of breast cancer due to mammography screening in women aged 40 to 49 in the United Kingdom
}

\author{
Necdet B Gunsoy ${ }^{1 *}$, Montserrat Garcia-Closas ${ }^{1,2}$ and Sue M Moss ${ }^{3}$
}

\begin{abstract}
Introduction: Overdiagnosis of breast cancer due to mammography screening, defined as the diagnosis of screendetected cancers that would not have presented clinically in a women's lifetime in the absence of screening, has emerged as a highly contentious issue, as harm caused may question the benefit of mammographic screening. Most studies included women over 50 years old and little information is available for younger women.
\end{abstract}

Methods: We estimated the overdiagnosis of breast cancer due to screening in women aged 40 to 49 years using data from a randomised trial of annual mammographic screening starting at age 40 conducted in the UK. A sixstate Markov model was constructed to estimate the sensitivity of mammography for invasive and in situ breast cancer and the screen-detectable mean sojourn time for non-progressive in situ, progressive in situ, and invasive breast cancer. Then, a 10-state simulation model of cancer progression, screening, and death, was developed to estimate overdiagnosis attributable to screening.

Results: The sensitivity of mammography for invasive and in situ breast cancers was 90\% (95\% Cl, 72 to 99$)$ and $82 \%$ (43 to 99), respectively. The screen-detectable mean sojourn time of preclinical non-progressive and progressive in situ cancers was 1.3 (0.4 to 3.4) and 0.11 (0.05 to 0.19) years, respectively, and 0.8 years (0.6 to 1.2) for preclinical invasive breast cancer. The proportion of screen-detected in situ cancers that were non-progressive was 55\% (25 to 77) for the first and 40\% (22 to 60) for subsequent screens. In our main analysis, overdiagnosis was estimated as $0.7 \%$ of screen-detected cancers. A sensitivity analysis, covering a wide range of alternative scenarios, yielded a range of $0.5 \%$ to $2.9 \%$.

Conclusion: Although a high proportion of screen-detected in situ cancers were non-progressive, a majority of these would have presented clinically in the absence of screening. The extent of overdiagnosis due to screening in women aged 40 to 49 was small. Results also suggest annual screening is most suitable for women aged 40 to 49 in the United Kingdom due to short cancer sojourn times.

\section{Introduction}

Since the introduction of mammography screening in many countries, a substantial increase in the incidence of breast cancers has been observed, raising concern about the potential for overdiagnosis of breast cancer due to screening. However, no consensus has been reached on the extent of such overdiagnosis. An overdiagnosed breast cancer is defined as one which is screen-detected, and would never have presented clinically in a woman's

\footnotetext{
* Correspondence: necdet.gunsoy@icr.ac.uk

'Division of Genetics and Epidemiology, Institute of Cancer Research, 15 Cotswold Road, Sutton, SM2 5NG, UK

Full list of author information is available at the end of the article
}

lifetime in the absence of screening [1]. In addition to overdiagnosis and consequent overtreatment, screening results in additional years lived with breast cancer due to the advancement of time of diagnosis. Estimates of overdiagnosis in previous studies vary considerably. Comparisons of expected breast cancer incidence extrapolated from rates before the introduction of screening with that observed after have resulted in estimates of overdiagnosis ranging from $4 \%$ [2] to 52\% [3] of all diagnosed breast cancers. Variations between estimates reflect the methodological challenges faced when estimating overdiagnosis. A drop in breast cancer incidence is observed in the age group immediately above that invited for screening due
C Biomed Central 
to the advancement of diagnosis of these cases by screening. Studies that do not account for this compensatory drop tend to have a higher estimate of overdiagnosis. Estimates will also vary depending on whether or not in situ cancers are included [1,4].

Simulation modelling is a popular tool for estimating the extent of overdiagnosis due to screening; it requires estimates of the mean duration of pre clinical cancer states (mean sojourn time), the screening test sensitivity (STS), and the background incidence of breast cancer in the absence of screening. De Koning et al. applied this approach to Dutch screening data for women aged 50 to 74 , and estimated that $3 \%$ of all cancers and $8 \%$ of screen-detected cancers were overdiagnosed [5].

Most estimates of overdiagnosis are based on data for women aged 50 years and over, as younger women are currently not eligible for screening in most countries. The extension of the age range of screening programmes to include younger women is under debate, but little is known on the extent of overdiagnosis due to screening in these women. Also, evidence suggest that younger women tend to have breast cancers that progress faster and lower mammography STS, mostly due to higher breast density, than older women [6-9], which may be favourable with regards to overdiagnosis.

In this study, we model data from a trial of mammographic screening for breast cancer starting at age 40 (Age trial) conducted in the UK, using data collected from the start of the trial in 1991 until 31st December 2010 , in order to estimate, in women aged 40 to 49 :

1. the STS of mammography for invasive and in situ breast cancers, that is the probability of a mammographic screen detecting a cancer that is in the preclinical state,

2. the mean sojourn time (MST), that is the mean duration, in years, for a cancer from first becoming detectable by screening to clinical diagnosis, of the screen-detectable preclinical breast cancer states: progressive in situ, non-progressive in situ, and invasive,

3. the proportion of screen-detected in situ cancers that are non-progressive,

4. the proportion of breast cancers diagnosed that would not have presented clinically in the absence of screening after accounting for a compensatory drop in incidence.

\section{Materials and methods}

Data

Details of the Age trial are given elsewhere [10]. In summary, a randomised controlled trial was designed to assess the effectiveness of annual screening by mammography in women from age 40 onwards in the UK. The trial comprised of an intervention arm of 53,890 women assigned to annual screening invitation and a control arm of 106,971 women not offered screening. Recruitment began in 1991 and the trial included 23 centres. Women were invited each year, except for those who specified that they did not wish to participate in the trial. Two-view mammography was performed on the first attendance to screening. The following screens were single view unless otherwise indicated. All diagnosed breast cancers, including interval, screen-detected, and those in the control arm, were recorded and submitted to a pathologic review.

Women with diagnosed breast cancer at entry to the trial were excluded from this analysis. Out of the 53,890 women assigned to the intervention arm, 36,348 attended the first screen and were eligible for analysis. For each following screen, women were included only if they attended all previous screening rounds. Only screening episodes recorded from ages 40 to 49 years as part of the trial were considered, and the analysis was limited to the first eight screening invitations since few women received more than this number. The exact date of each screen and of any breast cancer diagnosis was known for each woman. Interval cancers were defined as cancers diagnosed up to 12 months after a negative routine screen, and time since the previous negative screen was calculated in months. In all eight screening rounds, a total of 194 screen-detected, and 122 interval cancers were recorded. The numbers of women screened, and of screen-detected and interval cancers in each screening round are presented for in situ and invasive cancers in Table 1.

The Age trial is registered as an International Standard Randomised Controlled Trial, number 24647151. Ethical approval was obtained for the trial from London MREC (MREC/98/2/40), and NGIB (formerly PIAG) approval (PIAG 3-07(h)/2002) was obtained for the use of identifiable patient information.

\section{Statistical methods}

We constructed two Markov models to estimate the extent of overdiagnosis (Figure 1): one to estimate screening parameters based on the Age trial data (parameter estimation model), and one to estimate overdiagnosis based on parameter estimates from the first model (overdiagnosis model).

\section{Parameter estimation model}

We constructed a six-state Markov model similar to that in a previous study [11]; states included were healthy, screen-detectable non-progressive in situ (NPIS), clinically diagnosed non-progressive in situ (CIS), screendetectable progressive in situ (PIS), screen-detectable preclinical invasive breast cancer (PIBC), and clinically diagnosed invasive breast cancer (CIBC). 
Table 1 Cancer detection by screening round in a trial of annual mammographic screening starting age 40, UK.

\begin{tabular}{|c|c|c|c|c|c|c|c|}
\hline \multirow[t]{2}{*}{ Screening round } & \multirow[t]{2}{*}{ Women screened $^{1}$} & \multicolumn{2}{|c|}{$\begin{array}{l}\text { Screen-detected } \\
\text { cancers }\end{array}$} & \multicolumn{2}{|c|}{$\begin{array}{c}\mathrm{ICs}^{2} \text { in the first } 12 \\
\text { months }\end{array}$} & \multirow[t]{2}{*}{ Mean age (years) } & \multirow[t]{2}{*}{ Mean screening interval (years) } \\
\hline & & $\mathrm{INV}^{3}$ & $1 S^{4}$ & $\mathrm{INV}^{3}$ & $1 S^{4}$ & & \\
\hline 1 & 36348 & 31 & 6 & 7 & 2 & 40.5 & - \\
\hline 2 & 30779 & 20 & 3 & 17 & 2 & 41.6 & 1.1 \\
\hline 3 & 27083 & 16 & 3 & 17 & 0 & 42.6 & 1.1 \\
\hline 4 & 24188 & 15 & 5 & 17 & 1 & 43.7 & 1.1 \\
\hline 5 & 21107 & 16 & 4 & 10 & 0 & 44.7 & 1.0 \\
\hline 6 & 18603 & 13 & 7 & 16 & 1 & 45.6 & 1.0 \\
\hline 7 & 17193 & 19 & 9 & 18 & 1 & 46.6 & 1.0 \\
\hline 8 & 13998 & 21 & 6 & 11 & 2 & 47.5 & 0.9 \\
\hline Total & 189299 & 151 & 43 & 113 & 9 & 43.4 & \\
\hline
\end{tabular}

${ }^{1}$ Only includes women screened at all previous rounds; ${ }^{2}$ interval cancer; ${ }^{3}$ invasive carcinomas; ${ }^{4}$ in-situ carcinomas.

Several important assumptions were made on the natural history of breast cancer in order to simplify the model specification. First, we assumed that there were two types of in situ lesions: one that would progress into invasive cancer, and another that would never progress into invasive cancer, but could become clinically detected in the absence of screening. Thus, PIS would not be diagnosed in the absence of screening and would eventually progress into PIBC before becoming clinically diagnosed. Also, in situ cancers detected at screening would be either from PIS or NPIS, whereas those observed in the screening interval or in the absence of screening would be exclusively from NPIS. Second, we assumed that all PIBC have a mandatory PIS precursor. Finally, we assumed that preclinical cancers could not regress, but only remain in their current state or progress.

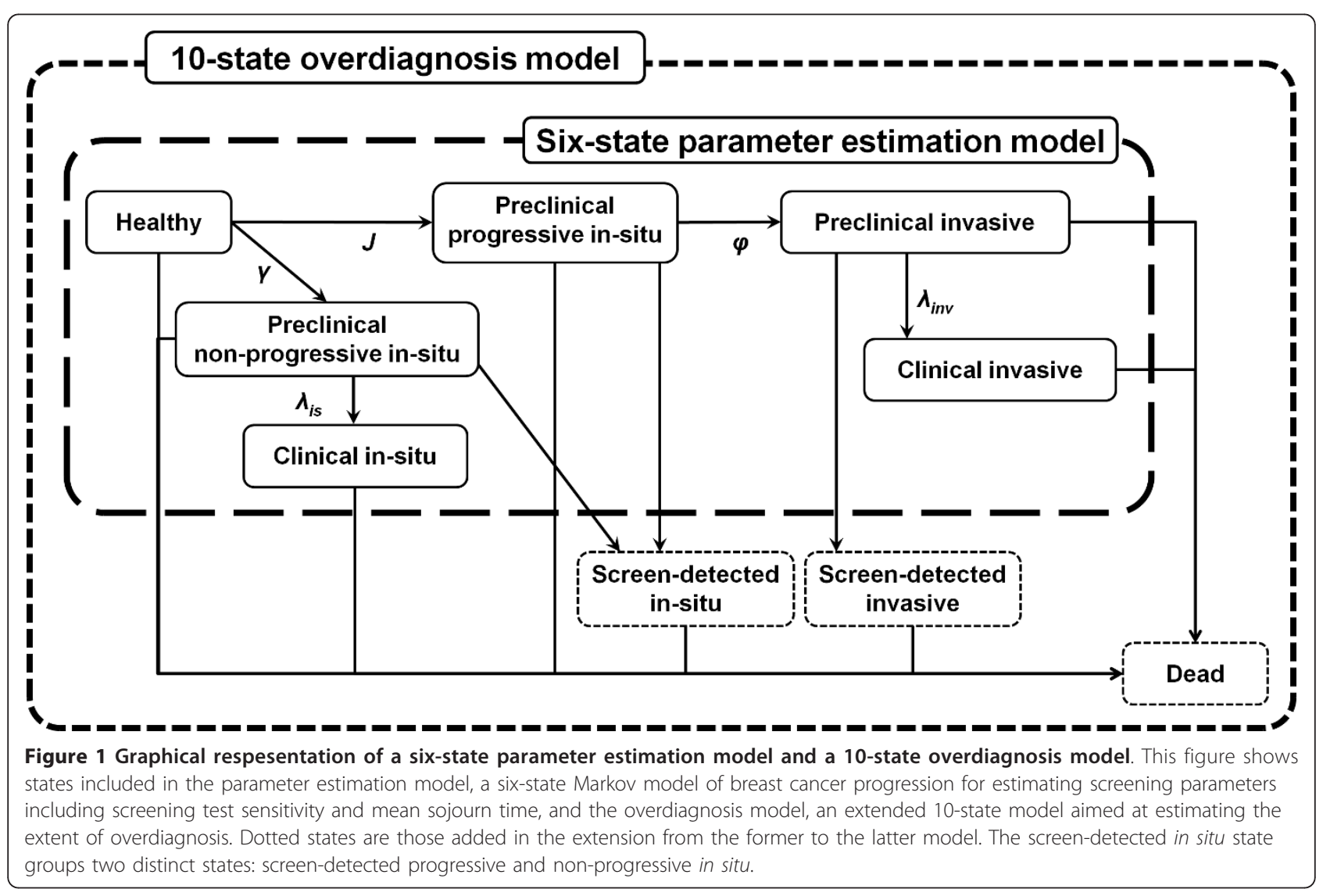


The intensity matrix, $Q$, for this model, with $J$, the background incidence of invasive breast cancer, $\gamma$, the background incidence of in situ breast cancer, $\varphi$, the transition rate between PIS and PIBC, $\lambda_{i s}$, the transition rate between NPIS and CIS, and $\lambda_{i n v}$, the transition rate between PIBC and CIBC was determined as

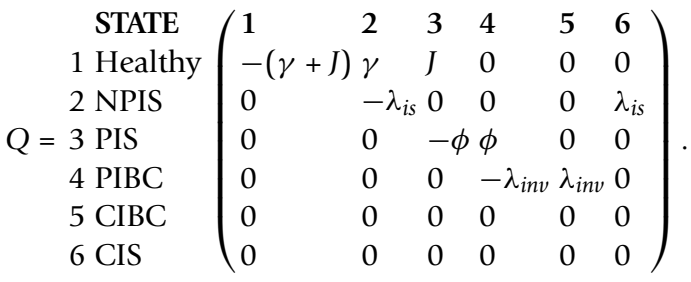

Both $J$ and $\gamma$ were obtained directly from the observed age-specific incidence in the control arm of the Age trial who were not offered any screening between the ages of 40 to 49 years. Given the assumption of exponential distribution of time to transition, the MST in a state was the inverse of the transition rate. From the intensity matrix, the probability of progression from any state $i$ to any state $j$ in any time interval $t$, can be defined as $P_{i j}(t)$. The derivation of transition probabilities is based on the solution of Kolmogorov equations and exponential distribution properties and will not be developed here [11-13]. Given transition probabilities, $P_{i j}(t)$, the probability of having a positive or negative mammogram, as well as the incidence of breast cancer in the interval between two screens can be formulated (Table 2).

Women in this analysis were free from diagnosed breast cancer at entry, meaning that, at the prevalent screen, probabilities were conditional on being healthy or in a preclinical disease state. The number of women screened in each round, $\mathrm{n} . \mathrm{scr}_{k}$, is given in Table 1 . The STS for in situ and invasive cancer were defined as $S_{i s}$ and $S_{i n v}$, respectively. In each screen, we defined the probability of screen detection of NPIS, PIS, and PIBC. The model was fitted to the observed number of in situ and invasive cancers detected in each screen. For incident screens, we defined the probability of having a false negative result in the previous screen for each preclinical cancer state. The monthly incidence of interval cancers was defined for CIS and CIBC and fitted to the observed incidence of in situ and invasive cancers, respectively, in the first 12 months after each screen. This analysis was performed using WinBUGS14. The median and 95\% credible interval (CI) of the posterior distribution for each

Table 2 Probability of cancer detection at prevalent and incident screens, and monthly incidence of interval cancers.

\begin{tabular}{|c|c|c|}
\hline Description & Parameter & Definition \\
\hline \multicolumn{3}{|l|}{ Any screen $k$} \\
\hline Number of women screened & n.scr ${ }_{k}$ & see Table 1 \\
\hline Negative screening episodes & n.neg ${ }_{k}$ & n.scr ${ }_{k}\left(1-\right.$ P.scr $_{n p i s_{k}}-$ P.scr $_{p i s_{k}}-$ P.scr $\left._{i n v_{k}}\right)$ \\
\hline \multicolumn{3}{|c|}{ Proportion of false negatives at screen $k$} \\
\hline False negative NPIS & P.fneg ${ }_{n p i s}$ & $\left(\mathrm{P} \mathrm{scr}_{n p i s_{k}} \mathrm{n} \mathrm{scr}_{k}\left(1-S_{i s}\right) / S_{i s}\right) /$ n.neg $_{k}$ \\
\hline False negative PIS & P.fneg $\operatorname{pis}_{h}$ & 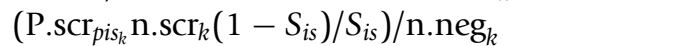 \\
\hline False negative PIBC & P.fneg inv $_{k}$ & $\left(\mathrm{P} \mathrm{scr}_{i n v_{k}} \mathrm{n}_{\mathrm{scr}}\left(1-S_{i n v}\right) / S_{i n v}\right) / \mathrm{n} . \mathrm{neg}_{k}$ \\
\hline Any pre-cancer state & P.fneg $k$ & P.fneg $_{n p i s_{k}}+$ P.fneg pis $_{k}+$ P.fneg inv $_{k}$ \\
\hline \multicolumn{3}{|l|}{ Prevalent screen } \\
\hline Screen-detected NPIS & P.scr $_{n p i s}$ & $S_{i s} P_{12}$ (age) $/\left(P_{11}\right.$ (age $)+P_{12}$ (age $)+P_{13}$ (age $)+P_{14}($ age $\left.)\right)$ \\
\hline Screen-detected PIS & P.scr ${ }_{p i s}$ & $S_{i s} P_{13}($ age $) /\left(P_{11}\right.$ (age $)+P_{12}$ (age $)+P_{13}($ age $)+P_{14}($ age $\left.)\right)$ \\
\hline Screen-detected PIBC & P.scr $_{i n v_{1}}$ & $S_{\text {inv }} P_{14}($ age $) /\left(P_{11}\right.$ (age $)+P_{12}$ (age $)+P_{13}$ (age $)+P_{14}($ age $\left.)\right)$ \\
\hline \multicolumn{3}{|l|}{ Incident screen $k$} \\
\hline Screen-detected NPIS & P.scr $_{n p i s_{k}}$ & $S_{i s}\left(\left(1-\right.\right.$ P.fneg $\left.{ }_{k-1}\right) P_{12}(t)+$ P.fneg fnis $\left._{k-1} P_{22}(t)\right)$ \\
\hline Screen-detected PIS & P.scr pis $_{k}$ & $S_{i s}\left(\left(1-\right.\right.$ P.fneg $\left.{ }_{k-1}\right) P_{12}(t)+$ P.fneg $\left.{ }_{n p i s_{k-1}} P_{22}(t)\right)$ \\
\hline Screen-detected PIBC & P.scr inv $_{k}$ & $S_{i n v}\left(\left(1-\right.\right.$ P.fneg $\left._{k-1}\right) P_{14}(t)+$ P.fneg $_{p i s_{k-1}} P_{34}(t)+P$. fneg $\left._{i n v_{k-1}} P_{44}(t)\right)$ \\
\hline \multicolumn{3}{|c|}{ Monthly incidence of interval cancers in the first $m$ months after screen $k$} \\
\hline \multirow[t]{3}{*}{ In-situ } & \multirow{3}{*}{$\mathrm{IC}_{i s_{k}}$} & n. $\operatorname{neg}_{k}\left(P_{11}(m-1) P_{16}(1)+P_{12}(m-1) P_{26}(1)\right)$ \\
\hline & & 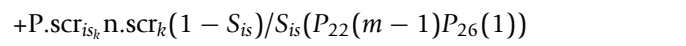 \\
\hline & & n.neg ${ }_{k}\left(P_{11}(m-1) P_{15}(1)+P_{13}(m-1) P_{35}(1)+P_{14}(m-1) P_{45}(1)\right)$ \\
\hline \multirow[t]{2}{*}{ Invasive } & \multirow{2}{*}{$\mathrm{IC}_{i n v_{k}}$} & $+{ }^{P} . \mathrm{scr}_{i n v_{k}} \mathrm{n} . \mathrm{scr}_{k}\left(1-S_{i n v}\right) / S_{i n v}\left(P_{44}(m-1) P_{45}(1)\right)$ \\
\hline & & + P.scr $\operatorname{scisk}_{k} \mathrm{n} . \mathrm{scr}_{k}\left(1-S_{i s}\right) / S_{i s}\left(P_{34}(m-1) P_{45}(1)+P_{33}(m-1) P_{35}(1)\right)$ \\
\hline
\end{tabular}


parameter was obtained through Gibbs sampling, using 5 chains of 3000 iterations. Due to their correlated nature, we also estimated the correlation between MST and STS.

\section{Overdiagnosis model}

The first model was extended to include 10 states in order to estimate the absolute amount of overdiagnosis due to screening (Figure 1). States included healthy, preclinical NPIS, preclinical PIS, PIBC, screen-detected NPIS, screen-detected PIS, CIS, screen-detected PIBC, CIBC, and dead. In the simulation, 1,000,000 women were followed up for 15 years in monthly cycles starting from age 40. Transitions probabilities between states were calculated using data from various sources: (1) the Office for National Statistics (ONS), for the incidence of invasive and in situ breast cancer [14] and all-cause death rates [15] in women aged 40 to 54 from 2008, (2) the Age trial [10], for the incidence of invasive and in situ breast cancer in women aged 40 to 49 , (3) the parameter estimation model in this study, for the STS and MST in pre-cancer states in women aged 40 to 49 , and (4) estimates from previous studies $[7,8,11]$ for the MST in women aged over 50 . For the breast cancer incidence in women aged 40 to 44 , we used incidence rates reported by ONS. For women aged 44 to 49 , ONS incidence rates are affected by screening at age 49 ; we therefore used the incidence in the control arm of the Age trial, adjusted by the ratio of the ONS rates to Age trial rates for the 40 to 44 age group. This resulted in higher incidence rates than those observed in the Age trial control arm; it was therefore not necessary to adjust these rates for selection bias due to the lower observed rate in non-attenders. For the base-case analysis, the medians of our MST and STS estimates were used. We also performed sensitivity analyses to investigate the impact of changing MST and STS on the estimate of overdiagnosis. We considered the following scenarios: high MST, low MST, high STS, low
STS, high MST with low STS, and high MST with high STS (Table 3). This analysis was performed using TreeAge Pro 2011 (TreeAge Software Inc., Williamstown, MA, USA).

\section{Results}

Estimates from the parameter estimation model are shown in Table 4. The median and 95\% CI for the invasive and in situ mammography STS were $90.0 \%$ (72.0 to 98.9$)$ and 81.7\% (43.4 to 99.0), respectively. Model estimates for the MST in the screen-detectable PIBC state was 0.84 years (0.64 to 1.21 ), which, added to the MST in the screendetectable PIS state, 0.11 years ( 0.05 to 0.19$)$, gave a mean window of 0.95 years for a cancer to be detected via screening before arising clinically. For screen-detectable NPIS, the MST was 1.29 years (0.41 to 3.44 ). The estimated proportion of screen-detected in situ cancers that were non-progressive was 55\% (25-77) in the prevalent and $40 \%$ (22 to 60) in incident screens.

Results of the overdiagnosis model are given in Table 5 . In our base-case analysis, 16,030 breast cancers were diagnosed between the ages of 40 and 49 years in women offered screening, in contrast with 15,425 in women not offered any screening, a surplus of 605 cases, equivalent to $6.2 \%$ of screen-detected and $3.8 \%$ of all cases. However, in ages 50 to 54 , where screening is not offered in both simlulated groups, 541 additional cases were diagnosed in women not offered screening previously, resulting in a total of 64 overdiagnosed cases equivalent to $0.7 \%$ of screen-detected cases and $0.4 \%$ of all cancers diagnosed within ages 40 to 49 years.

Estimates of overdiagnosis in our sensitivity analysis ranged from 0.5 to $2.9 \%$ of screen-detected cancers and $0.3 \%$ to $2.2 \%$ of all cancers diagnosed within ages 40 to 49 years. The highest impact on overdiagnosis was observed when increasing the MST, whereas increasing the STS had a smaller impact on overdiagnosis (Table 6).

Table 3 Parameter definitions for base-case and sensitivity analyses of overdiagnosis model.

\begin{tabular}{|c|c|c|c|c|c|c|c|c|}
\hline \multirow[b]{3}{*}{ Invasive } & \multirow{2}{*}{\multicolumn{2}{|c|}{$\begin{array}{l}\text { Screening test } \\
\text { sensitivity (\%) }\end{array}$}} & \multicolumn{6}{|c|}{ Mean sojourn time (years) } \\
\hline & & & \multicolumn{3}{|c|}{40 to 49} & \multicolumn{3}{|c|}{$50-59$} \\
\hline & Invasive & In-situ & NPIS & PIS & PIBC & NPIS & PIS & $\overline{\text { PIBC }}$ \\
\hline Base case & \multicolumn{2}{|c|}{ Median $^{1}$} & \multicolumn{3}{|c|}{ Median } & 3 & 0.175 & 1.825 \\
\hline Long MST & \multicolumn{2}{|c|}{ Median } & \multicolumn{3}{|c|}{ Upper limit $^{2}$} & 5 & 0.25 & 2.75 \\
\hline Short MST & \multicolumn{2}{|c|}{ Median } & \multicolumn{3}{|c|}{${\text { Lower } \text { limit }^{3}}^{3}$} & 2 & 0.1 & 1.5 \\
\hline High sensitivity & 100 & 100 & & & & 3 & 0.175 & 1.825 \\
\hline Low sensitivity & \multicolumn{2}{|c|}{ Lower limit } & \multicolumn{3}{|c|}{ Median } & 3 & 0.175 & 1.825 \\
\hline Low sensitivity, long MST & \multicolumn{2}{|c|}{ Lower limit } & \multicolumn{3}{|c|}{ Upper limit } & 5 & 0.25 & 2.75 \\
\hline High sensitivity, long MST & 100 & 100 & \multicolumn{3}{|c|}{ Upper limit } & 5 & 0.25 & 2.75 \\
\hline
\end{tabular}

${ }_{1,2,3}$ Refers to the median, upper limit, and lower limit of results from parameter estimation model, respectively. MST, median sojourn time; NPIC, non-progressive in situ; PIBC, preclinical invasive breast cancer; PIS, preclinical progressive in situ. 
Table 4 Model estimates of breast cancer screening and progression parameters in women aged 40 to 49 years

\begin{tabular}{|c|c|c|}
\hline \multirow[t]{2}{*}{ Parameter } & \multicolumn{2}{|c|}{ Estimate } \\
\hline & Median & $95 \% \mathrm{Cl}$ \\
\hline \multicolumn{3}{|c|}{ Screening test sensitivity (\%) } \\
\hline Invasive & 90.0 & $(72.0-98.9)$ \\
\hline In-situ & 81.7 & $(43.4-99.0)$ \\
\hline \multicolumn{3}{|c|}{ Mean sojourn time (years) } \\
\hline Invasive & 0.84 & $(0.64-1.21)$ \\
\hline Progressive in situ & 0.11 & $(0.05-0.19)$ \\
\hline Non-progressive in situ & 1.29 & $(0.41-3.44)$ \\
\hline \multicolumn{3}{|c|}{$\%$ of screen-detected in situ that is non-progressive } \\
\hline Prevalent screen & 55 & $(25-77)$ \\
\hline Incident screens & 40 & $(22-60)$ \\
\hline
\end{tabular}

Model fit

When compared to data from the Age trial, the parameter estimation model accurately predicted the screendetected invasive cancers for the first six screens, but underestimated those for the last two screens (Table 7). For screen-detected in situ cancers, model predictions were accurate for the first five screens, but underestimated the observed number for the last three screens. The number of invasive interval cancers were overestimated for the first screen, and underestimated in the last three screens. The expected number of in situ interval cancers were underestimated for the last screen only. For all screens combined, the model slightly underestimated the number of screen-detected invasive cancers. Expected values from the overdiagnosis models were within the range of estimates from the parameter

Table 5 Comparison of the number of cancers detected for 1,000,000 women in annual screening between ages 40 to 49 years versus no screening versus.

\begin{tabular}{|c|c|c|c|c|c|c|}
\hline & \multicolumn{2}{|c|}{ Ages 40 to 49} & \multicolumn{2}{|c|}{ Ages 50 to 54} & \multicolumn{2}{|c|}{ Ages 40 to 54} \\
\hline & $\begin{array}{l}\text { Group } 1 \text { Annual } \\
\text { screening }\end{array}$ & $\begin{array}{l}\text { Group } 2 \text { No } \\
\text { screening }\end{array}$ & $\begin{array}{l}\text { Group } 1 \text { No } \\
\text { screening }\end{array}$ & $\begin{array}{l}\text { Group } 2 \text { No } \\
\text { screening }\end{array}$ & $\begin{array}{c}\text { Group } 1 \\
\text { Total }\end{array}$ & $\begin{array}{c}\text { Group } 2 \\
\text { Total }\end{array}$ \\
\hline \multicolumn{7}{|c|}{ Base-case } \\
\hline \multicolumn{7}{|l|}{ Screen-detected } \\
\hline Invasive & 7772 & - & - & - & 7772 & - \\
\hline Progressive in situ & 1145 & - & - & - & 1145 & - \\
\hline Non-progressive in situ & 874 & - & - & - & 874 & - \\
\hline \multicolumn{7}{|l|}{ Clinically detected } \\
\hline Invasive & 5693 & 14089 & 7680 & 8151 & 13373 & 22240 \\
\hline Non-progressive in situ & 546 & 1336 & 658 & 728 & 1204 & 2064 \\
\hline All cancers & 16030 & 15425 & 8338 & 8879 & 24368 & 24304 \\
\hline \multicolumn{7}{|l|}{ Overdiagnosis } \\
\hline Absolute number & \multicolumn{2}{|c|}{605} & \multicolumn{2}{|c|}{-541} & \multicolumn{2}{|c|}{64} \\
\hline$\%$ of screen-detected & \multicolumn{2}{|c|}{6.2} & & & \multicolumn{2}{|c|}{0.7} \\
\hline $\begin{array}{r}\% \text { of cancers diagnosed within } \\
\text { ages } 40 \text { to } 49\end{array}$ & \multicolumn{2}{|c|}{3.8} & & & \multicolumn{2}{|c|}{0.4} \\
\hline
\end{tabular}

Estimates from the overdiagnosis model.

Table 6 Overdiagnosis of breast cancer due to annual screening in women aged 40 to 49 years.

\begin{tabular}{ccc}
\hline & Overdiagnosis & \\
\hline Scenario & \% of screen-detected & \% diagnosed within ages $\mathbf{4 0}$ to $\mathbf{4 9}$ \\
\hline Base-case & 0.7 & 0.4 \\
\hline Long MST & 2.7 & 2.0 \\
\hline Short MST & 0.5 & 0.3 \\
\hline High sensitivity & 0.7 & 0.4 \\
\hline Low sensitivity & 0.5 & 0.3 \\
\hline High sensitivity, long MST & 2.9 & 2.2 \\
\hline Low sensitivity, long MST & 2.7 & 1.6 \\
\hline
\end{tabular}


Table 7 Fit of model estimates to data observed in a trial of annual mammographic screening starting age 40 in the UK.

\begin{tabular}{|c|c|c|c|c|c|c|}
\hline \multirow[b]{3}{*}{$\begin{array}{r}\text { Screening } \\
\text { episode }\end{array}$} & \multicolumn{3}{|c|}{ Invasive } & \multicolumn{3}{|c|}{ In-situ } \\
\hline & Observed & \multicolumn{2}{|c|}{ Expected } & \multirow{2}{*}{$\begin{array}{c}\text { Observed } \\
\text { AGE trial }\end{array}$} & \multicolumn{2}{|c|}{ Expected } \\
\hline & AGE trial & $\begin{array}{c}\text { Paremeter estimation } \\
\text { model }\end{array}$ & $\begin{array}{l}\text { Overdiagnosis } \\
\text { model }\end{array}$ & & $\begin{array}{c}\text { Paremeter estimation } \\
\text { model }\end{array}$ & $\begin{array}{r}\text { Overdiagnosis } \\
\text { model }\end{array}$ \\
\hline \multicolumn{7}{|c|}{ Intervention arm (offered annual screening) } \\
\hline \multicolumn{7}{|c|}{ Screen-detected cancers } \\
\hline 1 & 31 & $28(22-34)$ & 32 & 6 & $7(3-12)$ & 7 \\
\hline 2 & 20 & $18(16-20)$ & 19 & 3 & $5(2-8)$ & 5 \\
\hline 3 & 16 & $17(15-19)$ & 18 & 3 & $5(2-7)$ & 5 \\
\hline 4 & 15 & 17(15-18) & 17 & 5 & $5(2-6)$ & 5 \\
\hline 5 & 16 & 15(13-17) & 16 & 4 & $5(2-6)$ & 4 \\
\hline 6 & 13 & $14(13-15)$ & 15 & 7 & $3(2-6)$ & 4 \\
\hline 7 & 19 & $14(12-15)$ & 14 & 9 & $3(2-6)$ & 4 \\
\hline 8 & 21 & $11(10-12)$ & 12 & 6 & $3(2-4)$ & 3 \\
\hline Total & 151 & 134(116-150) & 143 & 43 & $36(17-55)$ & 37 \\
\hline \multicolumn{7}{|c|}{ Interval cancers } \\
\hline 1 & 7 & $19(16-23)$ & 16 & 2 & $1.4(0.6-2.6)$ & 2 \\
\hline 2 & 17 & 17(14-19) & 14 & 2 & $1.3(0.5-2.4)$ & 1 \\
\hline 3 & 17 & 15(13-18) & 13 & 0 & $1.2(0.5-2.2)$ & 1 \\
\hline 4 & 17 & $13(11-15)$ & 13 & 1 & $1(0.4-1.9)$ & 1 \\
\hline 5 & 10 & $12(10-14)$ & 12 & 0 & $0.9(0.4-1.7)$ & 1 \\
\hline 6 & 16 & $12(10-13)$ & 11 & 1 & $0.9(0.4-1.7)$ & 1 \\
\hline 7 & 18 & $10(8-11)$ & 11 & 1 & $0.7(0.3-1.4)$ & 1 \\
\hline 8 & 11 & $4(3-5)$ & 9 & 2 & $0.2(0.1-0.5)$ & 1 \\
\hline Total & 113 & 102(85-118) & 99 & 9 & $7.6(3.2-14.4)$ & 9 \\
\hline \multicolumn{7}{|c|}{ Control arm (not offered screening) } \\
\hline 40 & 52 & -1 & 69 & 1 & - & 5 \\
\hline 41 & 115 & - & 111 & 8 & - & 7 \\
\hline 42 & 115 & - & 124 & 7 & - & 9 \\
\hline 43 & 129 & - & 133 & 7 & - & 12 \\
\hline 44 & 138 & - & 147 & 15 & - & 13 \\
\hline 45 & 161 & - & 155 & 16 & - & 12 \\
\hline 46 & 161 & - & 160 & 12 & - & 10 \\
\hline 47 & 165 & - & 165 & 7 & - & 11 \\
\hline 48 & 172 & - & 166 & 13 & - & 12 \\
\hline Total & 1208 & - & 1230 & 86 & - & 91 \\
\hline
\end{tabular}

${ }^{1}$ The parameter estimation model was built using the incidence in the control arm.

estimation model, and had a similar fit to the observed data in the intervention arm. The fit of the expected numbers of cancers in the control arm was good, with a slight overprediction overall of approximately $2 \%$.

\section{Discussion}

In this study, we aimed to quantify the overdiagnosis of breast cancer attributable to screening women aged 40 to 49 years annually by first estimating screening parameters in a six-state Markov model using data from a trial of annual mammographic screening starting age 40 conducted in the UK. In women aged 40 to 49 years in the UK, we estimated that only $0.3 \%$ to $2.2 \%$ of all cancers were overdiagnosed.

An implicit assumption of the Markov process is that the time to transition is distributed exponentially. This distribution has been used in many instances previously and has been shown to have a good fit to progression models 
for breast cancer, as well as other cancers $[11,12,16]$. We assumed that all in situ cancers that arose in the absence of screening were non-progressive. In reality, a proportion of in situ cases detected in the absence of screening may be progressive. However, this proportion could not be estimated when relaxing this assumption.

Estimates from our overdiagnosis model suggest a 5\% reduction in the detection of invasive cancers as a result of screening. Previously, three screening trials (the TwoCounty, Stockholm, and Goteborg trials) showed a non-significant reduction of $5 \%$ to $10 \%$ in the incidence of invasive breast cancer when comparing the incidence in the screened and control groups [17]. Also, we assumed that all invasive cancers had an in situ precursor, which may not be the case [18]. Hence, our model may have overestimated the number of invasive cancers detected in their in situ precursor state. However, it is unlikely that this would affect our estimates of overdiagnosis as both in situ and invasive breast cancers were included in our calculations.

Our results showed that the STS for in situ cancers was approximately $10 \%$ lower than for invasive cancers, probably due to the larger size of invasive tumours [19]. Although no previous studies estimated the STS of in situ cancers separately, previous estimates of the STS for preclinical breast cancer ranged from $69 \%$ to $100 \%$ [6-8,13], consistent with our 90\% STS estimate for PIBC. In the Age trial, two-view mammograms were performed at prevalence screen, and one-view at incident screens unless indicated otherwise. When estimated separately, we found a $10 \%$ difference in prevalent (95\%, 79 to 100) and incident STS (85\%, 69 to 95). However, this model did not show any improvement in fit, and could not estimate the difference in STS for in situ and invasive breast cancer, which are more important parameters with regards to overdiagnosis due to screening. In addition, the STS of mammography is likely to increase with increasing age [9]; it was not possible to incorporate this in the current model, but our sensitivity analysis found that increasing the sensitivity had limited impact on the estimate of overdiagnosis. Estimates of MST and STS are necessarily related. The correlation between STS and MST in our model was 0.75 and their distribution showed no sign of bimodality. The sensitivity analysis addressed the correlated nature of STS and MST, by the inclusion of a scenario with long MST and small STS as an alternative to our base-case model, which had short MST and high STS.

The MST for screen-detectable NPIS was the longest among pre-cancer states, suggesting that more NPIS are detected in the prevalent screen than in incident screens. For screen-detectable PIS, the MST was very short, roughly three to ten weeks. Being much shorter than the yearly screening interval, this implies that few progressive lesions are detected in the in situ stage. However, the pool of progressive lesions will renew itself at each screen, implying that the rate of progressive lesions detected at each screen is constant proportionally to the background incidence of invasive breast cancer. According to our estimates, the combined MST of PIS and PIBC was under one year in $66 \%$ of cases. This would support annual screening for women aged 40 to 49. Biennial or triennial screening would result in many women developing both pre-cancer and having a clinical diagnosis during the screening interval.

To our knowledge, only one study used a six-state Markov model to estimate the detection rates of NPIS in screening, but did not estimate STS [11]. Using UK data for women aged 50 and over, authors predicted that $39 \%$ and $21 \%$ of screen-detected in situ cancers were non-progressive at prevalent and incident screens, respectively. The model had a lack of fit for UK data, overestimating cancers detected at prevalent and underestimating cancers detected at incident screens. In this study, we predicted a higher proportion of NPIS, possibly due to a higher relative background incidence of $i n$ situ to invasive cancer in women aged 40 to 49 compared to women aged 50 to 69 [14]. In previous studies, the MST of PIBC in women aged 40 to 49 ranged from 1.05 to 2.46 years $[6-8,13,20]$, which is longer than our estimate of 0.95 years. However, this study is the first to report MST estimates for women aged 40 to 49 in the UK, and for older women, previous estimates show a shorter MST in British women compared to other European countries [11].

Our estimate of overdiagnosis for annual screening in women aged 40 to 49 in the UK was in line with those reported in other studies. Hellquist et al. [21] estimated that $1 \%$ ( -6 to 8$)$ of all breast cancers were overdiagnosed in a screening programme for women aged 40 to 49 screened every 18 months in Sweden. In a systematic review, the range of overdiagnosis for women aged 40 to 49 years was $-4 \%$ to $7.1 \%$ [22]. Despite large credible intervals in our estimates of STS and MST, the range of overdiagnosis from this study was small, $0.3 \%$ to $2.2 \%$ of breast cancers diagnosed within ages 40 to 49 years. Thus, although precise estimates of STS and MST are hard to obtain, the estimate of overdiagnosis is relatively unaected. Our sensitivity analysis included a large range of STS and MST values, and our results should be generalisable to other countries with similar breast cancer incidence rates as the UK. However, it is not clear to what extent our results are extendable to programmes with longer screening intervals: the impact of screening frequency on overdiagnosis in women aged 40 to 49 years would require further studies. 


\section{Conclusions}

The most important implication of this study is that, in women aged 40 to 49 in the UK, a small proportion of breast cancers were overdiagnosed due to screening, between $0.3 \%$ to $2.2 \%$ of all breast cancers diagnosed within ages 40 to 49 years. Since women aged 40 to 49 have shorter MST, lower STS, and lower mortality rates than women aged 50 and over, less overdiagnosis would normally be expected which may explain why estimates of overdiagnosis from this study are smaller than those reported for women aged 50 onwards [2,3,23,24]. Second, although a high proportion of in situ cancers detected at screening were estimated to be non-progressive, the great majority of these would have presented clinically in the absence of screening, implying they would not be overdiagnosed. Finally, the mean sojourn time of preclinical invasive breast cancer, including its in situ precursor, was just under one year, suggesting that annual screening would be most appropriate for women aged 40 to 49 .

\section{Abbreviations \\ CIBC: clinical invasive breast cancer; CIS: clinical in situ; MST: mean sojourn time; NPIS: non-progressive in situ; PIBC: preclinical invasive breast cancer; PIS: preclinical progressive in situ; STS: screening test sensitivity.}

\section{Authors' contributions}

NBG participated in the conception and design of the study, the development of the methodology and the interpretation of results, performed the analyses, and drafted the manuscript. MGC participated in the conception of the study, the interpretation of results and reviewed the manuscript. SMM participated in the conception and design of the study, the acquisition of data, the development of the methodology and the interpretation of results, revised and reviewed the manuscript, and supervised the study. All authors read and approved the final manuscript.

\section{Competing interests}

The authors declare that they have no competing interests.

\section{Acknowledgements}

NBG is supported by the Institute of Cancer Research. MGC is supported by Breakthrough Breast Cancer Research. The Age trial was supported by grants from the Medical Research Council and Cancer Research UK, and also received funding from the Department of Health and the US National Cancer Research Institute.

\section{Author details}

${ }^{1}$ Division of Genetics and Epidemiology, Institute of Cancer Research, 15 Cotswold Road, Sutton, SM2 5NG, UK. ²Breakthrough Breast Cancer Centre, Institute of Cancer Research, 237 Fulham Road, London, SW3 6JB, UK. ${ }^{3}$ Centre for Cancer Prevention, Queen Mary University of London, Wolfson Institute of Preventive Medicine, Charterhouse Square, London, EC1M 6BQ, UK.

Received: 27 June 2012 Accepted: 29 November 2012 Published: 29 November 2012

\section{References}

1. Puliti D, Miccinesi G, Paci E: Overdiagnosis in breast cancer: design and methods of estimation in observational studies. Prev Med 2011, 53:131-133.

2. Duffy SW, Tabár L, Olsen AH, Vitak B, Allgood PC, Chen THH, Yen AMF, Smith RA: Absolute numbers of lives saved and overdiagnosis in breast cancer screening, from a randomized trial and from the Breast Screening Programme in England. J Med Screen 2010, 17:25-30.

3. Jørgensen KJ, Gøtzsche PC: Overdiagnosis in publicly organised mammography screening programmes: systematic review of incidence trends. BMJ 2009, 339:b2587.

4. de Gelder R, Heijnsdijk EAM, van Ravesteyn NT, Fracheboud J, Draisma G, de Koning HJ: Interpreting overdiagnosis estimates in population-based mammography screening. Epidemiol Rev 2011, 33:111-121.

5. de Koning HJ, Draisma G, Fracheboud J, de Bruijn A: Overdiagnosis and overtreatment of breast cancer: microsimulation modelling estimates based on observed screen and clinical data. Breast Cancer Res 2006, 8:202.

6. Tabár L, Fagerberg G, Chen HH, Duffy SW, Smart CR, Gad A, Smith RA: Efficacy of breast cancer screening by age. New results from the Swedish Two-County Trial. Cancer 1995, 75:2507-2517.

7. Duffy SW, Day NE, Tabár L, Chen HH, Smith TC: Markov models of breast tumor progression: some age-specific results. J Natl Cancer Inst Monogr 1997, 22:93-97.

8. Brekelmans CT, Westers P, Faber JA, Peeters PH, Collette HJ: Age specific sensitivity and sojourn time in a breast cancer screening programme (DOM) in The Netherlands: a comparison of different methods. J Epidemiol Community Health 1996, 50:68-71.

9. Carney PA, Miglioretti DL, Yankaskas BC, Kerlikowske K, Rosenberg R, Rutter CM, Geller BM, Abraham LA, Taplin SH, Dignan M, Cutter G, BallardBarbash R: Individual and combined effects of age, breast density, and hormone replacement therapy use on the accuracy of screening mammography. Ann Intern Med 2003, 138:168-75.

10. Moss S: A trial to study the effect on breast cancer mortality of annual mammographic screening in women starting at age 40. Trial Steering Group. J Med Screen 1999, 6:144-148.

11. Yen MF, Tabár L, Vitak B, Smith RA, Chen HH, Duffy SW: Quantifying the potential problem of overdiagnosis of ductal carcinoma in situ in breast cancer screening. Eur J Cancer 2003, 39:1746-1754.

12. Duffy SW, Chen HH, Tabár L, Day NE: Estimation of mean sojourn time in breast cancer screening using a Markov chain model of both entry to and exit from the preclinical detectable phase. Stat Med 1995, 14:1531-1543.

13. Duffy SW, Chen HH, Tabár L, Fagerberg G, Paci E: Sojourn time, sensitivity and positive predictive value of mammography screening for breast cancer in women aged 40-49. Int J Epidemiol 1996, 25:1139-1145.

14. Office for National Statistics: Cancer Statistics: Registrations. Registrations of cancer diagnosed in 2008, England. Series MB1 No. 39. 2008 [http:// www.statistics.gov.uk/downloads/theme health/mb1-39/mb1-no39-2008. pdf].

15. Office for National Statistics: Mortality statistics: Deaths registered in 2008. Review of the National Statistician on deaths in England and Wales, 2008. Series DR. 2008 [http://www.statistics.gov.uk/downloads/theme health/DR2008/DR 08.pdf].

16. Prevost TC, Launoy G, Duffy SW, Chen HH: Estimating sensitivity and sojourn time in screening for colorectal cancer: a comparison of statistical approaches. Am J Epidemiol 1998, 148:609-619.

17. Moss S: Overdiagnosis and overtreatment of breast cancer: overdiagnosis in randomised controlled trials of breast cancer screening. Breast Cancer Res 2005, 7:230-234.

18. Sinn HP: Breast cancer precursors: lessons learned from molecular genetics. J Mol Med (Berl) 2009, 87:113-115.

19. Weedon-Fekjaer $\mathrm{H}$, Tretli S, Aalen OO: Estimating screening test sensitivity and tumour progression using tumour size and time since previous screening. Stat Methods Med Res 2010, 19:507-527.

20. Paci E, Duffy SW: Modelling the analysis of breast cancer screening programmes: sensitivity, lead time and predictive value in the Florence District Programme (1975-1986). Int J Epidemiol 1991, 20:852-858.

21. Hellquist BN, Duffy SW, Nystrm L, Jonsson $\mathrm{H}$ : Overdiagnosis in the population-based service screening programme with mammography for women aged 40 to 49 years in Sweden. J Med Screen 2012, 19:14-19.

22. Biesheuvel C, Barratt A, Howard K, Houssami N, Irwig L: Effects of study methods and biases on estimates of invasive breast cancer overdetection with mammography screening: a systematic review. Lancet Oncol 2007, 8:1129-1138.

23. Moss S, Thomas I, Evans A, Thomas B, Johns L: Randomised controlled trial of mammographic screening in women from age 40: results of screening in the first 10 years. Br J Cancer 2005, 92:949-954. 
24. Olsen AH, Agbaje OF, Myles JP, Lynge E, Duffy SW: Overdiagnosis, sojourn time, and sensitivity in the Copenhagen mammography screening program. Breast J 2006, 12:338-342.

doi:10.1186/bcr3365

Cite this article as: Gunsoy et al: Modelling the overdiagnosis of breast cancer due to mammography screening in women aged 40 to 49 in the United Kingdom. Breast Cancer Research 2012 14:R152.

Submit your next manuscript to BioMed Central and take full advantage of:

- Convenient online submission

- Thorough peer review

- No space constraints or color figure charges

- Immediate publication on acceptance

- Inclusion in PubMed, CAS, Scopus and Google Scholar

- Research which is freely available for redistribution

Submit your manuscript at www.biomedcentral.com/submit 